\title{
The Use of Probiotic with ORS and ORS Only in Children with Acute Diarrhea
}

\author{
Rida Ali \\ Department of Paediatrics, Bahria Medical University, Karachi, Pakistan
}

\begin{abstract}
Objective: To compare the mean number of stools per day in children treated with combination of probiotic (lactobacillus rhamnosus) with ORS and ORS only in acute diarrhea.

Study Design: Randomised control trial.

Place and Duration of Study: Department of Pediatric Medicine, PNS Shifa Hospital, from February to July, 2017.

Methodology: A total of 80 children with acute watery diarrhea were randomly divided into two groups. Forty patients in first group were given probiotic with ORS and 40 patients in second group (control) were given ORS only. All children were monitored from 0 day (inclusion day) to next 5 days. Demographic data was collected regarding age, gender, weight and frequency of loose stools. Dehydration status was also assessed at the time of admission by the attending physician. Data was collected through a structured proforma.

Results: The average age of the children was $24.3 \pm 18.65$ months. There were $47(58.8 \%)$ males and $33(41.3 \%)$ females. Mean number of stools was significantly low in those patients who were treated with combination of probiotic (lactobacillus rhamnosus) with ORS than those who were treated with ORS only in acute diarrhea $(3.25 \pm 1.13$ vs. $4.13 \pm 0.79 ; p<0.001)$. Conclusion: Probiotics are found to be significantly more effective in reducing the stool frequency in acute diarrhea.
\end{abstract}

Key Words: Acute diarrhea, Probiotic, ORS, Lactobacillus rhamnosus (LGG), Saccharomyces boulardii (SB), Randomised controlled trials (RCTs, non-RCTs).

How to cite this article: Ali R. The use of probiotic with ORS and ORS only in children with acute diarrhea. J Coll Physicians Surg Pak 2019; 29(12):1179-1182.

\section{INTRODUCTION}

Among 5-year old children, diarrheal diseases remain the second leading cause of mortality worldwide. Diarrheal diseases remain the leading cause in more than 2.5 billion episodes of illness and 1.5 million annual deaths globally in developing countries ( 500 deaths per day in Pakistan). ${ }^{1}$ On average every child suffers from 56 episodes of diarrhea per year in Pakistan. ${ }^{2}$ The prevalence of diarrhea in Punjab was found as $7.8 \%{ }^{3}$

Rowland et al. brought out the consensus that the efficacy of probiotics were species and often strain specific, in reducing the incidence and duration of rotavirus diarrhea in infants, clostridium difficile infections, symptomatic improvements in irritable bowel syndrome, alleviation of digestive discomfort, inflammatory bowel disease, respiratory or genitourinary infections, helicobactorpylori infection, and atopic dermatitis have also shown reduced frequency and severity of necrotising enterocolitis in premature infants; and especially regulate intestinal immunity. ${ }^{4}$

Correspondence to: Dr. Rida Ali, Bahria Medical University,

DHA Phase 2, Karachi, Pakistan

E-mail:drrida.ali09@gmail.com

Received: January 28, 2019; Revised: June 21, 2019;

Accepted: October 04, 2019
European Society for Paediatric Gastroenterology, Hepatology and Nutrition (ESPGHAN) 2014, based on systemic review highlights the use of different strains of probiotics like, lactobacillus rhamnosus GG, saccharomyces boulardii and lactobacillus reuteri DSM 17938 in addition to rehydration in children.5,6

A meta-analysis of RCTs and non-RCTs, found significantly reduced duration of diarrhea and stool frequency in $S$. boulardii group as compared to control. ${ }^{7}$ The duration of diarrhea was reduced in children treated with L. reuteri for 5 days as compared to control group ( 70.7 [SD 26.1] vs 103.8 [SD 28.47] hours, respectively; $\mathrm{p}<.001)$ in a single-blind clinical trial by Dinleyici et al.8 Lactobacillus rhamnosus was also shown to have reduced effects in duration of acute diarrhea that is 0.47 (Cl95\%:-0.80 to $-0.14 ; p=0.020$ ) in different randomised controlled trials. ${ }^{9}$

The study conducted by Aggarwal has shown significantly shorter duration of diarrhea, in LGG group [60 (54-72) $\mathrm{h}$ vs. 78 (72-90) $h ; P<0.001] .10$

The diarrhea related mortality and morbidity is very high in developing countries. This can be reduced by prompt treatment and management like ORS and probiotic. The literatures from developed countries have shown the effective use of probiotic in management of acute diarrhea. 
Since there is limited data available from Pakistan, the aim of the study was to compare the beneficial effect of probiotic use with ORS against ORS only in the management of acute diarrhea, so that probiotic with ORS can be used as an add-on therapy in management of acute diarrhea in children.

\section{METHODOLOGY}

This study, a randomised control trial, was conducted at Department of Pediatric Medicine, PNS Shifa Hospital from February to July-2017.

Acute diarrhea was defined as the presence of at least three evacuations of liquid or loose stools, within 24 hours, for a maximum period of 14 days. Degree of dehydration was assessed by WHO guidelines, as no dehydration (no sunken eyes, skin turgor $<2 \mathrm{sec}$ ); and two or more of the following signs for some dehydration: (restlessness, irritability, sunken eyes, drinks eagerly, skin pinch goes back slowly) on clinical examination. Outcome variables were assessed by mean number of stool for 5 days in both treatment groups.

The sample size was calculated by using OpenEpi Software taking mean number of stools per day at $5 \%$; $80 \%$ power of the test with $9.17 \pm 1.88$ in lactobacillus rhamnosus group and $10.36 \pm 1.87$ in control group. 10 The sample size was 80 children (40 in each group). The sample technique was consecutive non-probability. Children presented with acute watery diarrhea between 6 months to 5 years age of either gender were included.

All children with chronic diarrhea (presence of loose stools more than 14 days), coeliac disease confirmed by objective modality (such as physician record/hospital laboratory investigation), tropical sprue confirmed by objective modality (such as physician record/hospital laboratory investigation), infectious diarrhea confirmed by objective modality (such as physician record/hospital laboratory investigation), children with systemic complications, high fever, severe malnutrition, measles, pertussis and use of antidiarrheal confirmed by objective modality (such as physician record/hospital laboratory investigation) were excluded. Children on intravenous antibiotic confirmed by objective modality (such as physician record/hospital laboratory investigation), children on IV therapy for severe dehydration (two or more signs of lethargy or unconsciousness, sunken eyes, unable to drink or drink poorly, skin pinch goes back very slowly $>2$ seconds, according to the definitions of the WHO guidelines for the treatment of diarrhea) ${ }^{11}$ were not included.

Permissions from the concerned administrative authorities and Ethical Committee were taken. The risks and benefits of the study were explained to the parents, informed written consents were taken for their children examination and intervention according to the guidelines of Helsinki Declaration. Permission was also taken regarding use of data for research and publication. All children, fulfilling inclusion criteria, were included in the study.

Children were randomised into two groups by using sealed opaque envelope method. The process of randomisation was performed by the nursing staff (the nurse did not know details of participants in order to maintain the unpredictability of next assignment).

First group was given probiotic 5 billion CFU in sachet form twice a day for 5 days (orally administered diluted in water or mixed with semi-solid food) along with rehydration therapy (children with 'No Dehydration' were given ORS $100 \mathrm{ml}$ after each stool in <2 years of age and $200 \mathrm{ml}$ after each stool in $>2$ years of age, children with some dehydration were given ORS $75 \mathrm{ml} / \mathrm{kg}$ during first 4 hours). The second group (control) was given ORS only (children with "No Dehydration" were given ORS $100 \mathrm{ml}$ after each stool in <2 years of age and 200 $\mathrm{ml}$ after each stool in $>2$ years of age, children with some dehydration were given ORS $75 \mathrm{ml} / \mathrm{kg}$ during first 4 hours ). All children were monitored from 0 day (inclusion day) to next 5 days.

Demographic data was collected regarding age, gender, weight, frequency of loose stools; duration of diarrhea in days. Dehydration status was also assessed at the time of admission by attending physician. Appropriate entries and data were collected through a structured study proforma.

SPSS version 17 was used to enter and analyse the data. Descriptive statistics was used to analyse and describe data. Quantitative variables like age, weight, duration of diarrhea, number of stools per day mentioned as means and standard deviations. Qualitative variables like gender, dehydration presented as frequencies and percentages in both probiotic and control group. Mean number of stools was compared in both probiotic and control groups by using independent t-test, taking $p$-value $\leq 0.05$ as significant. Effect modifiers/confounders like age, weight, gender, duration of diarrhea dehydration were dealt through stratification. Post-stratification "t-test" was applied to see the effect of modifiers on outcome. The $p$-value $\leq 0.05$ was taken as significant.

\section{RESULTS}

The mean age between probiotic and ORS group was $15.60 \pm 14.191$ months vs. $33.00 \pm 18.652$ months. Mean number of stools in children was significantly low in those patients who were treated with combination of probiotic (lactobacillus rhamnosus) with ORS than those who were treated with ORS only in acute diarrhea [3.25 \pm 1.13 vs. $4.13 \pm 0.79 ; p<0.001]$. Stratification analysis was performed and it was observed that mean number of stools in children was significantly low in those patients who were treated with combination of probiotic (lactobacillus rhamnosus) with ORS than those who 
Table I: Comparison of mean number of stools in children between groups for each age strata groups.

\begin{tabular}{|c|c|c|c|c|c|c|c|}
\hline \multirow[t]{3}{*}{ Age groups (months) } & \multicolumn{6}{|c|}{ Mean number of stools } & \multirow[t]{3}{*}{$p$-value } \\
\hline & \multicolumn{3}{|c|}{ Probiotic group } & \multicolumn{3}{|c|}{ Control (ORS) group } & \\
\hline & $\mathrm{N}$ & Mean & Std. Deviation & $\mathrm{n}$ & Mean & Std. Deviation & \\
\hline$\leq 12$ months & 26 & 3.48 & 1.08 & 12 & 3.83 & 0.76 & 0.321 \\
\hline 12.1 to 36 months & 11 & 2.68 & 1.10 & 12 & 4.13 & 0.80 & 0.002 \\
\hline$>36$ months & 3 & 3.30 & 1.25 & 16 & 4.37 & 0.77 & 0.062 \\
\hline
\end{tabular}

were treated with ORS only in acute diarrhea in only age group 12.21 to 36 months, while in other age stratified groups, mean number of stools was not significant between groups (Table I).

\section{DISCUSSION}

Four billion diarrheal episodes occur every year that results in $4 \%$ of all deaths and $5 \%$ of these lost to disability world-wide. Impairment in intestinal absorption of both micronutrients and macronutrients, malnutrition, and growth faltering is mainly caused by acute diarrhea.12,13 Diarrhea is the leading cause for high mortality rate, approximately 1.4 million of the 9 million child deaths in 2008 and $49 \%$ of the mortality in five countries namely India, Nigeria, Democratic Republic of the Congo, Pakistan and China. ${ }^{14,15}$ As diarrhea is the leading cause of death in children in 2008, compared to AIDS, malaria and measles combined, vitamin A supplementation, immunisation, access to safe drinking water and exclusive breast feeding was a progressive step in prevention of diarrhea in some areas. 15,16 Evidence have shown that use of zinc ${ }^{17}$ and probiotics, either singly or in a combination, can effectively prevent diarrhea. Probiotics either monocultures or mixed cultures of live organisms have promising results in the host by improving the properties of indigenous microflora, hampering the growth of diarrheal pathogens, and boosting cellular and humoral immunity. ${ }^{18}$

In this study, mean number of stools in children was significantly low in those patients who were treated with combination of probiotic (lactobacillus rhamnosus) with ORS than whose who treated with ORS only in acute diarrhea supported by many international studies. Diarrhea was found to be reduced in a randomised double-blind controlled trial in infants (1-23 months) who received milk formula supplemented with bifidobacterium lactis daily for one week than controls. ${ }^{19}$

Children (6-120 months) with acute infectious diarrhea, have shown significant reduction in duration of diarrhea in the symbiotic and the zinc groups compared to control group $(91.0 \pm 28.9$ hours vs. $114.3 \pm 30.9$ hours, $p<0.001$; $86.4 \pm 30.8$ hours vs. $114.3 \pm 30.9$ hours, $p<0.001$, respectively) in a single-centre, randomised, clinical trial in Turkey. ${ }^{20}$ Reuteri given for 5 days compared with oral rehydration therapy alone (control group) have significantly reduced duration of diarrhea (approximately 15h, $60.4 \pm 24.5 \mathrm{~h}$ vs. $74.3 \pm 15.3 \mathrm{~h}),(p<0.05) .{ }^{21}$
However, a meta-analysis of 3 randomised controlled trials have also shown significantly reduced duration of diarrhea in probiotic group compared to placebo (mean difference [MD] -24.82 hours, $95 \% \mathrm{Cl}-38.8$ to -10.8$) .22$ Probiotic such as, saccharomyces boulardii group, has significantly higher efficacy rate $(45 \%)$ compared to lactic acid producing probiotic (26\%).23 This was also shown in reducing duration of diarrhea in children (3 months to 5 years) when administered (SB) for 5 days than control group. 24

Mean number of stools in children was significantly low in those patients who were treated with combination of probiotic (lactobacillus rhamnosus) with ORS than those who were treated with ORS only in acute diarrhea in only age group 12.21 to 36 months. In other age stratified groups, mean number of stools was not significant between groups. This observation is also supported in a randomised clinical trial at Cairo, in children (1-23 months) number of stools per day was less than in controls (3.96 \pm 0.62 vs. $4.46 \pm 0.85)(p=0.04) .19$ However, healthy and non-hospitalised infants 6-36 months with acute diarrhea treated with ORS enriched with lactobacillus reuteri DSM17938 and zinc also showed reduced diarrheal episodes on day 2 $(p<0.001) .25$ Additional studies would be beneficial to determine the efficacy of probiotic in relation to patients age as there is significant variation.

\section{CONCLUSION}

Probiotics are found to be effective in reducing the stool frequency in acute diarrhea. Probiotics have modest beneficial effects; and the anticipated advantage must be considered as effective available alternatives in acute diarrhea.

\section{ETHICAL APPROVAL:}

Permissions from the concerned administrative authorities and Ethical Committee were taken.

\section{PATIENTS' CONSENT:}

Informed consent was obtained from patients to publish the data concerning this case.

\section{CONFLICT OF INTEREST:}

Authors declared no conflict of interest.

\section{AUTHORS' CONTRIBUTION:}

RA: Drafted and finalised the manuscript. The author read and approved the final manuscript. 


\section{REFERENCES}

1. Bhutta ZA, Kailegman RM, Stanton BF, Geme JW, Schor NF, Behrman RE. Acute gastroenteritis in children. Nelson Textbook of Pediatrics. Ed. 19th, Philadelphia WB Saunders Company; 2011: p. 1323-5.

2. Billoo AG, Memon MA, Khaskheli SA, Murtaza G, Iqbal K, SaeedShekhani M, et al. Role of probiotics (S.boulardii) in management and prevention of diarrhea. World $J$ Clin Gastroenterol 2006; 12:4557-60.

3. Punjab Bureau of Statistics, Planning and Development Department, Government of Punjab. Multiple indicators cluster survey. Punjab; 2007-2008.

4. Rowland I, Capurso L, Collins K, Cummings J, Delzenne N, Goulet $\mathrm{O}$, et al. Current level of consensus on probiotic science report of an expert meeting London, 23 November 2009. Gut Microbes 2010; 1:436-9.

5. Guarino A, Ashkenazi S, Gendrel D, Lo Vecchio A, Shamir R, Szajewska $\mathrm{H}$, et al. European society for pediatric gastroenterology, hepatology, and nutrition / European society for pediatric infectious diseases evidence-based guidelines for the management of acute gastroenteritis in children in Europe: Update2014. J Pediatr Gastroenterol Nutr 2014; 59: 132-52.

6. Szajewska H, Guarino A, Hojsak I, Indrio F, Kolacek S, Shamir R, et al. Use of probiotics for management of acute gastroenteritis: A position paper by the ESPGHAN working group for probiotics and prebiotics. J Pediatr Gastroenterol Nutr 2014; 58:5319.

7. Feizizadeh S, Salehi-Abargouei A, Akbari V. Efficacy and safety of Saccharomyces boulardii for acute diarrhea. Pediatrics 2014; 134:e176-91.

8. Dinleyici EC; PROBAGE Study Group, Vandenplas Y. Lactobacillus reuteri DSM17938 effectively reduces the duration of acute diarrhoea in hospitalised children. Acta Paediatr 2014; 103:e300-5.

9. Ahmadi E, Alizadeh-Navaei R, Rezai MS. Efficacy of probiotic use in acute rotavirus diarrhea in children: A systematic review and meta-analysis. Caspian J Intern Med 2015; 6:187-95.

10. Aggarwal S, Upadhyay A, Shah D, Teotia N, Agarwal A, Jaiswal V. Lactobacillus GG for treatment of acute childhood diarrhoea an open labelled, randomized controlled trial. Indian J Med Res 2014; 139:379-85.

11. World Health Organization. The World Health Report. WHO, Geneva 1999.

12. Marteau PR. Probiotics in clinical conditions. Clin Rev Allergylmmunol 2002; 22:255-73.

13. Szajewska H, Mrukowicz JZ. Use of probiotics in children with acute diarrhea. Paediatr Drugs 2005; 7:111-22.
14. Black RE, Cousens S, Johnson HL, Lawn JE, Rudan I, Bassani DG, et al. Child health epidemiology reference group of WHO and UNICEF: Global, regional, and national caused of child mortality in 2008: A systematic analysis. Lancet 2010; 375:1969-87.

15. Wardlaw T, Salama P, Brocklehurst C, Chopra M, Mason E. Diarrhoea why children are still dying and what can be done. Lancet 2010; 375:870-2.

16. Santosham M, Chandran A, Fitzwater S, Fischer-Walker C, Baqui AH, Black R. Progress and barriers for the control of diarrhoeal disease. Lancet 2010; 376: 63-7.

17. Bhutta ZA, Bird SM, Black RE, Gardner JM. Therapeutic effects of oral zinc in acute and persistent diarrhea in children in developing countries pooled analysis of randomized controlled trials. Am J Clin Nutr 2000; 72:1516-22.

18. Surawicz CM. Probiotics, antibiotic associated diarrhoea and Clostridium difficilediarrhoea in humans. Best Pract Res Clin Gastroenterol 2003; 17:775-83.

19. El-Soud NH, Said RN, Mosallam DS, Barakat NA, Sabry MA. Bifidobacteriumlactisin treatment of children with acute diarrhea: A randomized double blind controlled trial. Open Access Maced J Med Sci 2015; 3:403-7.

20. Yazar AS, Güven S, Dinleyici EÇ. Effects of zinc or synbiotic on the duration of diarrhea in children with acute infectious diarrhea. Turk J Gastroenterol 2016; 27:537-40.

21. Dinleyici EC, Dalgic N, Guven S, Metin O, Yasa O, Kurugol Z, et al. Lactobacillus reuteri DSM 17938 shortens acute infectious diarrhea in a pediatric outpatient setting. $J$ Pediatr (Rio J) 2015; 91:392-6.

22. Urbanska M, Gieruszczak-Bialek D, Szajewska H. Systematic review with meta-analysis: Lactobacillus reuteri DSM 17938 for diarrhoeal diseases in children. Aliment Pharmacol Ther 2016; 43:1025-34.

23. Asmat S, Shaukat F, Asmat R, Bakhat HFSG, Asmat TM. Clinical efficacy comparison of saccharomyces boulardii and lactic acid as probiotics in acute pediatric diarrhea. $J$ Coll Physicians Surg Pak 2018; 28:214-7.

24. Das S, Gupta PK, Das RR. Efficacy and Safety of Saccharomyces boulardii in acute rotavirus diarrhea: Double blind randomized controlled trial from a developing country. $J$ Trop Pediatr 2016; 62:464-70.

25. Maragkoudaki M, Chouliaras G, Moutafi A, Thomas A, Orfanakou A, Papadopoulou A. Efficacy of an oral rehydration solution enriched with lactobacillus reuteriDSM 17938 and zinc in the management of acute diarrhoea in infants: A randomized, double-blind, placebo-controlled trial. Nutrients 2018; 10.pii:E1189. 